\title{
Too few trials or too few reported trials?
}

\author{
Andrés Viteri-García', Nicholas J. DeVito², Ben Goldacre ${ }^{2}$ \\ 1. Faculty of Health Sciences 'Eugenio Espejo'. Centro de Investigación en Salud Pública y Epidemiología Clínica (CISPEC). \\ Universidad UTE. \\ 2. EBM DataLab, University of Oxford.
}

Earlier this year on the $20^{\text {th }}$ anniversary of the Evidence-Based Dentistry journal editor Derek Richards argued that there have been too many systematic reviews, and not enough high-quality original research. ${ }^{1}$ We agree that more high quality primary research would be useful. But we would like to emphasise that there is a serious ongoing issue with the results of completed trials not being reported.

Publication and dissemination biases impact the complete assessment of evidence in medical research with numerous examples throughout the literature. ${ }^{2}$ Systematic reviews have shown that studies with statistically significant results are more likely to be published than those without significant findings. ${ }^{3,4}$ The reasons for non-publication are varied but include trialists reporting lack of time or low priority to report; feeling that results are not important enough; and journal rejection. ${ }^{5}$ Prior research has shown that results reporting on trial registries, such as ClinicalTrials.gov, is also poor. ${ }^{6-9}$

In January 2018 the first trials covered by updated US trial reporting regulations became due to report results. ${ }^{10}$ Under the Food and Drug Administration Amendments Act of 2007 (FDAAA) all 'applicable' clinical trials are required to report results directly onto the US government website ClinicalTrials.gov within one year of their primary completion date. The DataLab at the University of Oxford has launched the FDAAA TrialsTracker, a tool that publicly tracks the results status of each individual completed clinical trial covered by this law, using daily data imported from ClinicalTrials. gov. ${ }^{11}$ Trials are marked as 'reported' when they have submitted results, even if those results are not yet publicly available.

We searched the data from http://fdaaa.trialstracker.net to identify all trials in the oral health sciences which are due to report results under FDAAA. Among the 1248 studies identified (searched on 21 August 2018) 20 were related to dentistry. Of these, eight (40\%) are overdue to report. Of the unreported trials six (75\%) were sponsored by noncommercial educational organisations and two (25\%) were sponsored by pharmaceutical or biotechnology companies. Four (50\%) have a recruitment status discrepant with their listed primary completion date: the FDAAA law requires all trials past their provided primary completion date to report results regardless of trial status, on pain of a daily $\$ 11,569$ fine. Of the 12 reported trials, four trials (33\%) were reported late.

Short profiles of the unreported trials are included below:

NCT02387970: This trial compared outcomes for two different treatment protocols related to a new type of dental implant (NobelParallel CC implant). Fifty participants were expected to enroll. The primary completion date was estimated as June 2017, however the trial's status is currently 'recruiting'. This study was sponsored by Columbia University with information provided by the PI, Dr. Panos Papapanou.

NCT00820235: This trial evaluated the gum response to three different types of dental implants for the front upper jaw. One hundred and fifty-five participants were expected to enroll. The primary completion date was June 2017. The trial was sponsored by Dentsply Sirona Implants.

NCT01919515: This trial tested whether a new type of molar crown performed similarly to established stainless steel crowns. Fifty participants were expected to enroll. The primary completion date was estimated as June 2017, however the status of the study is 'unknown', meaning the completion date has passed and the record has not been verified in more than two years. The trial was sponsored by NuSmile, Ltd.

NCT02399228: This phase 2 trial examined a drug containing East Indian Sandalwood Oil for the treatment of oral mucositis. The trial had seven participants and the primary completion date was May 2017. The trial was sponsored by Santalis Pharmaceutical Inc. in collaboration with The University of Texas Health Science Center at San Antonio.

NCT02539342: This phase 3 trial was to examine if caphosol rinse prevents oral mucositis in children undergoing chemotherapy. A single participant was enrolled before the study terminated in March 2017. Terminated trials are required to disclose results on all recruited participants under FDAAA. The trial was sponsored by the Medical College of Wisconsin.

NCT03174678: This phase 4 trial examined the effect of dexmedetomidine treatment on preoperative cooperation and emergence delirium in young children undergoing complete dental treatment. The trial had 100 participants and primary completion date was March 2017. The trial was sponsored by Adnan Menderes University.

NCT03336853: This trial examined whether treatment with the HybenX device reduced bleeding during root canal treatment. Sixty participants were enrolled and the study reached its primary completion date on July 21 2017. The trial was sponsored by Dr. Riccardo Pace of the Azienda Ospedaliero-Universitaria Careggi.

NCT03033628: This trial aimed to evaluate whether the DentalVibe comfort system could be used to reduce pain during maxillary infiltration injection in paediatric dental patients. The primary completion date is listed as April 2017. This trial has an estimated enrollment of 21 participants and is sponsored by Cairo University.

Some of these trials may have reported their results elsewhere. For instance, NCT03174678 has published findings in BioMed Research International, ${ }^{12}$ however this does not meet the legal requirements 


\section{EDITORIAL RESPONSE}

of the FDAAA 2007. Previous research has shown that journal publications can fail to disclose important elements of methods and results, ${ }^{13}$ and that results reports on registries are more complete than journal publications of the same studies, especially with respect to adverse events. ${ }^{14,15}$

These unreported trials give important cause for reflection. New research can be informative for patient care. But clinical trials must be of high quality - with the results reported in full, and in a timely manner - to avoid wasting limited financial resources and patient goodwill.

\section{Acknowledges and Disclosures:}

Dr. Viteri-García is a Master candidate in Applied Clinical Research in Health Sciences at Autonomous University of Barcelona and is partially funded by a grant of Secretaria de Educación Superior Ciencia, Tecnología e Innovación - Ecuador.

Nicholas DeVito is a researcher and DPhil candidate at the EBM Datalab in the Nuffield Department of Primary Care Health Sciences, University of Oxford. He is employed under Dr. Goldacre's grant from the Laura and John Arnold Foundation and is supported in his studies through a studentship from the Naji Foundation.

Dr. Goldacre has received research funding from the Laura and John Arnold Foundation, the Wellcome Trust, the Oxford Biomedical Research Centre, the NHS National Institute for Health Research School of Primary Care Research, the Health Foundation, and the World Health Organization; he also receives personal income from speaking and writing for lay audiences on the misuse of science.
1. Richards D. Too many reviews too few trials. Evid Based Dent. 2018; 19: 2.

2. McGauran N, Wieseler B, Kreis J, Schüler YB, Kölsch H, Kaiser T. Reporting bias in medical research - a narrative review. Trials 2010; 11: 37.

3. Dwan K, Gamble C, Williamson P R, Kirkham JJ \& Reporting Bias Group. Systematic review of the empirical evidence of study publication bias and outcome reporting bias - an updated review. PLoS One 2013; 8: e66844.

4. Schmucker C, Schall LK, Portalupi S, et al. Extent of non-publication in cohorts of studies approved by research ethics committees or included in trial registries. PLoS One 2014; 9: e114023.

5. Song F, Parekh S, Hooper L, et al. Dissemination and publication of research findings: an updated review of related biases. Health Technol Assess 2010; 14: iii, ix-xi, 1-193.

6. Prayle AP, Hurley MN, Smyth AR. Compliance with mandatory reporting of clinical trial results on ClinicalTrials.gov: cross sectional study. BMJ 2012; 344: d7373.

7. Jones CW, Handler L, Crowell KE, Kell LG, (or Keil LG,) Weaver MA, Platts-Mills TF. Non-publication of large randomized clinical trials: cross sectional analysis. BMJ 2013; 347: f6104.

8. Anderson ML, Chiswell K, Peterson ED, Tasneem A, Topping J, Califf RM. Compliance with results reporting at ClinicalTrials.gov. N Engl J Med 2015; 372: 1031-1039.

9. Fain KM, Rajakannan T, Tse T, Williams RJ, Zarin DA. Results Reporting for trials with the same sponsor, drug, and condition in Clinicaltrials.gov and peer-reviewed publications. JAMA Intern Med 2018; 178: 990-992.

10. DeVito NJ, Bacon S, Goldacre B. FDAAA Trials Tracker: A live informatics tool to monitor compliance with FDA requirements to report clinical trial results. bioRxiv 266452 (2018). doi:10.1101/266452. Available at: https://www.biorxiv.org/ content/early/2018/03/12/266452)

11. Datalab, E. B. M. FDAAA TrialsTracker. FDAAA TrialsTracker (2018). Available at https://fdaaa.trialstracker.net/. (accessed June 2018)

12. Keles $S$, Kocaturk $O$. The effect of oral dexmedetomidine premedication on preoperative cooperation and emergence delirium in children undergoing dental procedures. Biomed Res Int 2017; 2017: 6742183.

13. Turner L, Shamseer L, Altman DG, Schulz KF, Moher D. Does use of the CONSORT Statement impact the completeness of reporting of randomised controlled trials published in medical journals? A Cochrane review. Syst Rev 2012; 1: 60.

14. Jones CW, Keil LG, Holland WC, Caughey MC, Platts-Mills TF. Comparison of registered and published outcomes in randomized controlled trials: a systematic review. BMC Med 2015; 13: 282

15. Riveros C, Dechartres A, Perrodeau E, Haneef R, Boutron I, Ravaud P.Timing and completeness of trial results posted at ClinicalTrials.gov and published in journals. PLoS Med 2013; 10: e1001566; discussion e1001566.

Evidence-Based Dentistry (2018) 19, 67-68. doi:10.1038/sj.ebd.6401317 